\title{
Gastrogastric Fistulas
}

\section{A Complication of Divided Gastric Bypass Surgery}

Sharon G. D. Cucchi, M.D., Walter J. Pories, M.D., Kenneth G. MacDonald, M.D., and Elizabeth J. Morgan, R.N.

From the Department of Surgery, East Carolina University School of Medicine, Greenville, North Carolina

\section{Objective}

This report warns that gastrogastric fistulas may follow the division of the stomach in bariatric surgery.

\section{Summary Background Data}

Although surgery is the most effective therapy for morbid obesity, the procedures are still undergoing evolution. One of the key elements in bariatric surgery is the partition of the stomach to develop a much smaller reservoir. The partition has been done with single layers of staples with almost universal failure and with double layers of staples with a failure rate of $11.8 \%$ when observed for a 12-year follow-up.

\section{Methods}

This report details the experience with a series of 100 consecutive patients in whom the partition was created by dividing the stomach.

\section{Results}

The course of six patients was complicated by gastrogastric fistulas. One of the patients had the gastric bypass as the initial bariatric operation; in the other five, the gastric bypasses were carried out to revise failed staple lines. Although one of the patients required drainage for a subphrenic abscess, two had only self-limited signs of infection. In the remaining three patients, there was no evidence of any complication.

\section{Conclusion}

Gastrogastric fistulas followed division of the stomach in $6 \%$ of our gastric bypass operations. Methods for avoiding this complication include oversewing staple lines, using strong bites of tissue during the anastomosis, avoiding obstruction of the Roux-en- $Y$ jejunal segment, and testing of the integrity of the anastomosis with methylene blue dyes. The ideal method for partition of the stomach remains to be developed.

Morbid obesity is a serious disease associated with diabetes mellitus, cardiovascular and pulmonary disease, musculoskeletal disorders, gallbladder disease, deep vein thrombosis, and venous stasis ulcers. ${ }^{1-3}$ The morbidly obese also suffer from psychological problems (depres- sion, anxiety, and low self-esteem) and discrimination in an appearance-conscious society that views obesity as less aesthetically pleasing. ${ }^{3,4}$ Many patients attempt diets and other weight-loss plans, which usually fail. A number of surgical procedures designed to create gastric re- 
striction or malabsorption have been developed and have become well-established alternatives to diets and other weight-loss plans. These procedures, however, are not without their complications. ${ }^{5,6}$

The roux-en-Y gastric bypass, a standard bariatric procedure, is the procedure of choice at our institution. This operation, although effective in creating significant weight reduction, has a documented $11.8 \%$ rate of stapleline dehiscence. ${ }^{7}$ These staple-line breakdowns result in failure of the procedure because the restrictive quality of the proximal pouch is no longer present. As the patient eats again without discomfort, and regains weight, revision of the failed operation remains the only option. Unfortunately, these revisions have an even higher rate of staple-line breakdowns than the initial operations. ${ }^{5,8}$

Two years ago, we modified our standard roux-en-Y gastric bypass, the Greenville gastric bypass to a divided gastric bypass (DGB) to avoid the $11.8 \%$ incidence of staple-line dehiscence. The DGB is performed in a similar manner to the Greenville gastric bypass; however, after formation of the proximal pouch, the stomach is divided between the double staple lines. We postulated that resultant separation of gastric pouches would free us of the dependence on staple lines and avoid this cause of failure.

Unfortunately, the new operation also has introduced a new complication: the formation of a fistula that communicates between the proximal gastric pouch and the distal gastric remnant. Fistula formation, although a commonly described phenomenon in various gastric operations and disease processus, rarely has been described in the realm of bariatric procedures. This retrospective study examines six patients with gastrogastric fistulas after DGB surgery at our institution.

\section{METHODS AND MATERIALS}

One hundred morbidly obese patients, each a minimum of 100 pounds over their ideal body weight as defined by the Metropolitan Insurance Tables of $1983,{ }^{9}$ have undergone the DGB at our institution by one of two surgeons (WJP and KGM). It was first performed in March 1985, for revision operations only, then in July 1990 , became our preferred primary procedure for both revision and initial bariatric operations.

Preoperative evaluation of patients for the DGB followed the same protocol as that for the Greenville gastric bypass. ${ }^{9}$ Patients were examined in our obesity clinic by one of the two surgeons and a nurse practitioner. Rou-

Address reprint requests to Dr. Walter J. Pories, M.D., Chairman, Department of Surgery, East Carolina University School of Medicine, Greenville, NC 27858.

Accepted for publication August 25, 1994. tine preoperative studies included electrolytes, complete blood count, urinalysis, arterial blood gasses, thyroidstimulating hormone, SMA-12 chemistries, glucose tolerance test, chest $\mathrm{x}$-ray, upper gastrointestinal series, pulmonary function studies, and a preoperative psychiatric evaluation. Further studies were performed, including echocardiograms and endoscopy, as indicated for individual patients.

The DGB is performed in a similar technique to the Greenville gastric bypass. ${ }^{9}$ After the formation of a small 30-mL proximal pouch with a TA-90 four-row stapling device, however, the stomach is divided between the two sets of staple lines by electrocautery. Each free end of the stomach subsequently is oversewn with an absorbable 30 synthetic suture. The $8-\mathrm{mm}$ roux-en-Y gastrojejunal anastomosis is constructed with two continuous 3-0 polypropylene sutures. After this, integrity of the proximal gastric staple line and the gastrojejunal anastomosis are determined intraoperatively by two methods. First, the abdominal cavity is filled with warm saline and watched for bubbles as air is injected through the nasogastric tube under pressure. Then methylene blue is introduced though the nasogastric tube, and the anastomosis is examined once again for leaks of the dye.

Postoperatively, patients are given nothing by mouth for approximately 3 days. Then they slowly are advanced to small feedings of Ensure Plus (Ross Laboratories, Columbus, $\mathrm{OH}$ ) three times a day. Before discharge, usually on the fifth or sixth postoperative day, radio-opaque upper gastrointestinal studies are ordered to examine for evidence of leaks on all patients. Evidence of a leak, a sustained tachycardia, high fever, or other signs of sepsis usually were considered indications for exploration, intensive lavage of the abdomen, and drainage of the leak.

Clinic charts and hospital records of those patients found to have formed gastrogastric fistulas were reviewed further and evaluated for similarities and possible etiologic agents.

\section{RESULTS}

Gastrogastric fistulas occurred in 6 of 100 patients who underwent the DGB at our institution since 1985. All six satisfied the criteria for the procedure; all were women between the ages of 29 and 67 years. One had the DGB as an initial operation; the remaining five operations were revisions.

These women demonstrated certain similarities in their hospital courses; they were each operated on by one surgeon (WJP), each had similar postoperative symptoms, and in all patients except one, a leak or abscess was discovered before the discovery of the gastrogastric fistula.

A review of these six cases and randomly selected pa- 
Table 1. POSTOPERATIVE SYMPTOMS

AND THEIR INCIDENCE IN SIX PATIENTS WITH GASTROGASTRIC FISTULAS

\begin{tabular}{lc}
\hline Symptom & Incidence \\
\hline Abdominal pain & 6 \\
Fever & 6 \\
Tachycardia & 6 \\
Nausea & 5 \\
Vomiting & 4 \\
Fatigue & 2 \\
Diarrhea & 2 \\
Shoulder pain & 1 \\
Tachypnea & 1 \\
Anorexia & 1 \\
\hline
\end{tabular}

tients of the other surgeon (KGM) demonstrated differences in the technique between the two surgeons of dividing the stomach, oversewing the staple lines, constructing the gastrojejunostomy, evacuation of the gastric pouch, and irrigation of the abdomen. Although both used the TA-90 stapling device, one (KGM) divided the stomach with a cautery whereas the other (WJP) used scissors. The former surgeon (KGM) constructed proximal gastric pouches that were about $10 \mathrm{~mL}$ larger than the latter (WJP), and the former surgeon's (KGM) jejunal tunnels through the mesocolon were large enough to admit three fingers. In oversewing the staple lines, this surgeon's (KGM) sutures were $2 \mathrm{~mm}$ apart, whereas the other surgeon's (WJP) were $4 \mathrm{~mm}$ apart. The former surgeon's (KGM) initial gastrostomies and jejunostomy openings were about $2 \mathrm{~mm}$ larger $(1 \mathrm{~cm} v s .8 \mathrm{~mm})$. Further, he (KGM) evacuated the proximal pouch of all blood before constructing the gastrojejunostomy, then irrigated the abdominal cavity with a bacitracin solution after completing the anastomosis.

Intraoperative assessment of staple line and anastomotic integrity was made in five of these patients by injecting air or methylene blue through the nasogastric tube and examining the proximal pouch for leaks. There were no intraoperative leaks discovered. This was true even for two patients whose cases were complicated by a malfunction of the stapling device, resulting in inadequate closure of the staple line. In both of these cases, the staple lines were carefully oversewn.

Table 1 shows a summary of the symptoms experienced postoperatively in these patients. All six patients had symptoms of fever, tachycardia, and abdominal pain. Less frequent symptoms included nausea, vomiting, fatigue, and diarrhea. Shoulder pain, tachypnea, and anorexia were each noted in one patient.

Table 2 shows the postoperative day each patient was diagnosed with either a leak or abscess and a fistula. Al- though three patients $(C, D, E)$ developed severe symptoms in the immediate postoperative period, requiring extended hospitalization, they were not diagnosed with a leak or abscess until postoperative day 6 , by a second, and this time, accurate meglumine diatrizoate (Gastrografin, Squibb Diagnostic Division, Princeton, NJ) swallow. One patient was returned to the operating room for drainage of her abscess. No abscess could be defined by computed tomography in the other two patients who were managed nonoperatively.

The three other patients $(\mathrm{A}, \mathrm{B}, \mathrm{F})$ were discharged within 6 days of their operation without evidence of complications. They each had developed a brief episode of fever or tachycardia during their postoperative hospitalization; however, septic work-ups failed to identify the source of their symptoms, which abated before discharge. Meglumine diatrizoate swallows were similarly normal. Within the first month after discharge, these three patients presented with complaints of worsening abdominal pain, nausea, vomiting, and fever. Further work-up with meglumine diatrizoate swallows at that time revealed either leak, abscess or fistula formation.

Three patients (A, B, C) were found to have postoperative stenosis or occlusion of the gastrojejunostomy before discovery of the gastrogastric fistula (Table 2).

\section{DISCUSSION}

Since institution of routine division of the stomach during gastric bypass (DGB) to avoid late staple line failures, we have encountered six patients of a series of 100 DGBs who developed fistulous communication between the proximal pouch and the distal stomach. Because the two portions of the stomach are completely separate, these were probably caused by a leak with abscess forma-

\section{Table 2. POSTOPERATIVE DAY EACH PATIENT WITH A GASTROGASTRIC FISTULA WAS DIAGNOSED WITH LEAK, STENOSIS, ABSCESS, AND FISTULA BY GASTROGRAFIN SWALLOW}

\begin{tabular}{ccccc}
\hline Patient & $\begin{array}{c}\text { Leak } \\
\text { Diagnosis } \\
\text { (POD) }\end{array}$ & $\begin{array}{c}\text { Anastomotic } \\
\text { Stenosis } \\
\text { (POD) }\end{array}$ & $\begin{array}{c}\text { Abscess } \\
\text { (POD) }\end{array}$ & $\begin{array}{c}\text { Gastrogastric } \\
\text { Fistula (POD) }\end{array}$ \\
\hline A & 23 & 95 & 23 & 133 \\
B & 22 & 4 & $*$ & 34 \\
C & 6 & 6 & $*$ & 27 \\
D & 6 & $*$ & $*$ & 9 \\
E & 6 & $*$ & & 63 \\
F & $*$ & $*$ & & \\
\end{tabular}




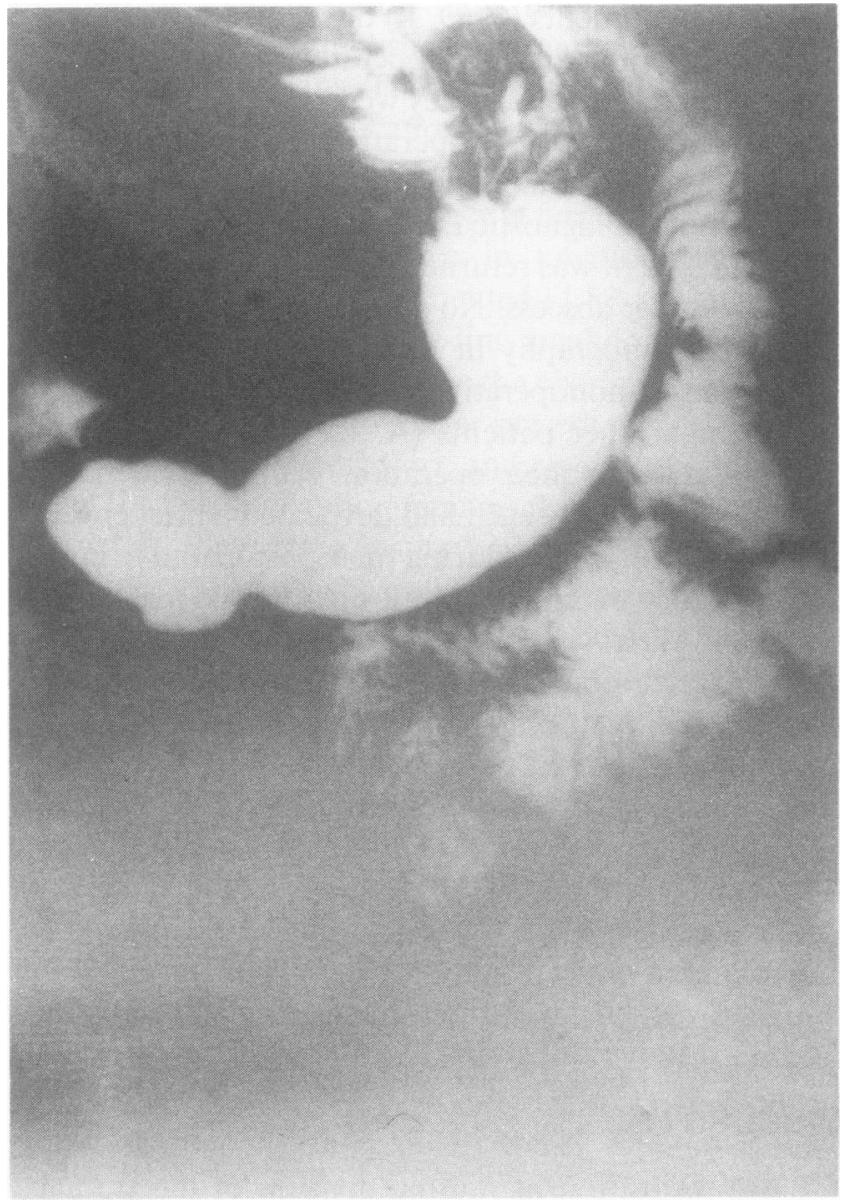

Figure 1. Gastrogastric fistula visualized by meglumine diatrizoate swallow. Contrast flows preferentially from proximal stomach to distal stomach via fistula.

tion adjacent to the distal staple line, which then drained into the distal stomach, forming the gastrogastric fistula (Fig. 1)..$^{10,11}$

In all cases, patients had either fever or tachycardia in the early postoperative course, although routine postoperative day 3 meglumine diatrizoate swallows were negative in each case. This fact emphasizes the well-known insensitivity of contrast studies in detecting leaks. Additional evaluation or treatment of suspected leaks in these patients often must be guided by their clinical presentations.

That five of six fistulas developed after revision operations is not surprising, because leaks and other complications are known to be more common with revision procedures. Dissection is much more difficult than with an initial operation, increasing the likelihood of injury to the stomach or its blood supply.

Three of the revision patients who developed leaks and gastrogastric fistulas also had postoperative stenosis or occlusion of the gastrojejunostomy. Distention of the gastric pouch secondary to an anastomotic stenosis could logically predispose to a leak from the divided staple line. Stenosis commonly arises in the first 2 months after surgery and complicates $2 \%$ to $19 \%$ of these operations. ${ }^{12}$ It can be minimized by construction of a larger anastomosis, but with an increased risk for less weight loss.

Our analysis of these patients indicates that surgical technique is an important etiology of gastric leak and subsequent fistula formation. The two surgeons performing the procedure at our institution developed their techniques of the DGB independently. After analysis of the different techniques used by them, the following recommendations were established to minimize complications:

1. Oversewing of the long divided staple lines of the proximal pouch and distal stomach with closely spaced sutures at $2 \mathrm{~mm}$ between stitches;

2. Construction of an 8- to $10-\mathrm{mm}$ anastomosis, taking care to include strong bites of both gastric and jejunal mucosa with every suture;

3. Avoidance of possible obstruction of the efferent jejunal limb by ensuring that the limb lies without tension and without kinks during its course for the transverse mesocolon to the gastric pouch;

4. Intraoperative tests of pouch and anastomosis integrity with air and methylene blue; from our results with these six patients, this obviously will not prevent all leaks, but hopefully will reduce the incidence.

Of particular interest is the fact that $50 \%$ of this group of were discharged from the hospital on a liquid diet on the sixth postoperative day. A gastric leak usually is a catastrophic complication, leading to sepsis and necessitating re-operation for drainage. By developing a fistula via the divided edge of the distal stomach, these patients probably internally drained their leaks or abscess, which explains their delayed presentation and absence of systemic sepsis.

Our current approach to these patients is to treat the abscess first by drainage and antibiotics. Even after late presentations with no apparent collection on imaging studies, a full therapeutic course of antibiotics would be recommended to treat local inflammation. Acid reduction with $\mathrm{H} 2$ blockers or omeprazole is recommended. Maintenance of adequate nutrition is very important and may be provided via the initial operation or by insertion of a jejunostomy tube.

The morbidity associated with these fistulas includes increased hospitalizations and costs, as well as risk from infectious complications. Long-term sequelae are unknown. Closure of the fistula has not been documented in any of the six patients. Two of the patients whose op- 
erations occurred 1.5 to 2 years ago have persistent fistulas with poor weight loss. The status of the fistula of the patient from 1985 is unknown, although she continues to have poor weight loss. Postoperatively at 6 months, two patients who currently have continued problems with abdominal pain and nausea and may come to reoperation.

Although we have recommended that the division of the stomach is preferable to staple partition, our incidence of gastrogastric fistulas in $6 \%$ gives us pause. That complication rate is better than the $11.8 \%$ staple-line dehiscence rate, but it still is too high. Accordingly, for primary operations, we currently are conducting a trial of Sugerman's $(H$. Sugerman, personal communication, 1992) approach of partitioning the stomach with three superimposed staple lines applied with the single row TA-90 stapling device. In spite of an extensive use of this approach, Sugerman maintains that he has seen very few staple-line failures. For revisions of failed procedures, however, the divided gastric bypass remains the procedure of choice and, for these patients, we have adopted the recommendations noted above. Thus far, we have no further gastrogastric fistulas, extraluminal leaks, or failures of the triple stapled partitions.

\section{References}

1. Deitel M, ed. Surgery for the Morbidly Obese Patient. Philadelphia: Lee and Febiger, 1989, p 22.

2. Deitel M, Shahi B. Morbid obesity: selection of patients for surgery. J Am Coll Nutr 1992; 11:457-462.

3. Griffen WO Jr. Bariatric surgery in the 1990's. Adv Surg 1992; 25 : 99-117.

4. Waters GS, Pories WJ, Swanson MS, et al. Long-term studies of mental health after the Greenville gastric bypass operation for morbid obesity. Am J Surg 1991; 161:154-157.

5. Linner JH, Drew RL. Reoperative surgery-indications, efficacy and long-term follow-up. Am J Clin Nutr 1992; 55:606s-610s.

6. Mason EE, Renquist KE, Jiang D. Perioperative risks and safety of surgery for severe obesity. Am J Clin Nutr 1992; 55:573s-576s.

7. Jordan CP, MacDonald KG, Pories WJ, et al. Staple line failure: an avoidable complication of the gastric bypass. Arch Surg 1995 (in press).

8. MacLean LD, Rhode BM, Forse RA. Late results of vertical banded gastroplasty for morbid and super obesity. Surgery 1990; 107:20-27.

9. Flickinger EG, Pories WJ, Meelheim HD, et al. The Greenville gastric bypass. Ann Surg 1984; 199:555-562.

10. Amlicke JA, Ponka JL. Gastrocolic and gastrojejunocolic fistulas: case of 16 cases. Am J Surg 1964; 107:744-750.

11. Prickett D, Montgomery R, Cheadle WG. External fistulas arising from the digestive tract. South Med J 1991; 84:736-739.

12. Sanyal AJ, Sugarman HJ, Kellum JM, et al. Stomal complications of gastric bypass: incidence and outcome of therapy. Am J Gastroenterol 1992; 87:1165-1169. 